\title{
BAND SURGERY ON KNOTS AND LINKS, III
}

\section{TAIZO KANENOBU}

\begin{tabular}{|c|l|}
\hline Citation & OCAMI Preprint Series \\
\hline Issue Date & 2015 \\
\hline Type & Preprint \\
\hline Textversion & Author \\
\hline Rights & $\begin{array}{l}\text { C } 2016 \text { World Scientific Publishing Company. For personal use only. No other } \\
\text { uses without permission. }\end{array}$ \\
\hline Relation & $\begin{array}{l}\text { Preprint of an article submitted for consideration in Journal of Knot Theory and } \\
\text { Its Ramifications } @ 2016 \text { World Scientific Publishing Company. } \\
\text { https://www.worldscientific.com/worldscinet/jktr. }\end{array}$ \\
\hline Is version of & https://doi.org/10.1142/S0218216516500565 \\
\hline
\end{tabular}

From: Osaka City University Advanced Mathematical Institute http://www.sci.osaka-cu.ac.jp/OCAMI/publication/preprint/preprint.html 


\title{
BAND SURGERY ON KNOTS AND LINKS, III
}

\author{
TAIZO KANENOBU
}

\begin{abstract}
We give two criteria of links concerning a band surgery: The first one is a condition on the determinants of links which are related by a band surgery using Nakanishi's criterion on knots with Gordian distance one. The second one is a criterion on knots with $H(2)$-Gordian distance two by using a special value of the Jones polynomial, where an $H(2)$-move is a band surgery preserving a component number. Then, we give an improved table of $H(2)$-Gordian distances between knots with up to seven crossings, where we add Zeković's result.
\end{abstract}

\section{INTRODUCTION}

Let $L$ be a link in $S^{3}$ and $b: I \times I \rightarrow S^{3}$ an embedding such that $L \cup b(I \times I)=b(I \times \partial I)$, where $I$ is the unit interval $[0,1]$. Then we may obtain a new link $M=(L \backslash b(I \times \partial I)) \cup$ $b(\partial I \times I)$, which is called a link obtained from $L$ by the band surgery along the band $B$, where $B=b(I \times I)$; see Fig. 1 . If $L$ and $M$ are oriented links, and a band surgery preserves the orientations of $L$ and $M$, the band surgery is said to be coherent, otherwise incoherent. If $L$ and $M$ are unoriented links, and a band surgery preserves the number of components, then it is called the $H(2)$-move.

Since any oriented link can be deformed into the trivial knot by a sequence of coherent band surgeries, we define the coherent band-Gordian distance between two oriented links $L$ and $M$ to be the least number of coherent band surgeries needed to deform $L$ into $M$, which we denote by $\mathrm{d}_{\mathrm{cb}}(L, M)$. Similarly, since any knot can be deformed into the trivial knot by a sequence of $H(2)$-moves, we define the $H(2)$-Gordian distance between two knots $J$ and $K$, which we denote by $\mathrm{d}_{2}(J, K)$. In particular, the $H(2)$-unknotting number of a knot $K, \mathrm{u}_{2}(K)$, is the $H(2)$-Gordian distance between $K$ and the trivial knot.

In this paper we give two criteria of links concerning a band surgery: The first one is a condition on the determinant of a knot or link which is obtained from an unknotting number one knot by a band surgery (Theorem 2.2). This is easily obtained by using a condition on the determinant of a knot obtained from an unknotting number one knot by a crossing change due to Nakanishi [10, 11] (Proposition 2.1). The idea of the proof is similar to that of Theorem 4.2 in [8], which gives a condition on the determinant of a link or knot obtained from a 2-bridge knot by a band surgery. This uses a condition on the determinant

Date: February 10, 2016.

1991 Mathematics Subject Classification. Primary 57M25; Secondary 57M27.

Key words and phrases. Band surgery, coherent band-Gordian distance, $H(2)$-unknotting number, $H(2)$ Gordian distance. 


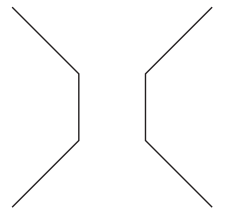

$L$

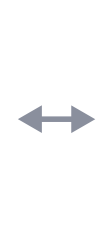

$L \cup B=M \cup B$

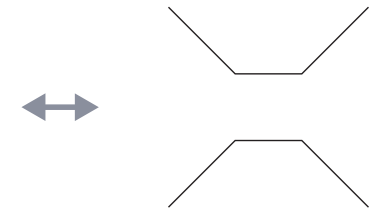

$M$

Figure 1 . The link $M$ is obtained from $L$ by a band surgery along the band $B$, and vice versa.

of a knot obtained from a 2-bridge knot by a crossing change due to Murakami [9]. Using Theorem 2.2 we give tables of the values for which the determinant of a link $L$ does not take such that either $\mathrm{d}_{2}(K, L)=1$ or $\mathrm{d}_{\mathrm{cb}}(K, L)=1$, where $K$ is an unknotting number one knot with determinant $\leq 115$ (Tables 1 and 2 ). They yield a table of pairs of an unknotting number one knot $J$ and a knot $K$ with $\mathrm{d}_{2}(J, K)>1$ (Table 3 ), and a table of pairs of an unknotting number one knot $J$ and a 2-component link $L$ with $\mathrm{d}_{\mathrm{cb}}(J, L)>1$, where the crossing numbers of $J, K$ and $L$ are $\leq 8$ (Table 4). As corollaries of Theorem 2.2, we obtain a condition for an unknotting number one knot to have $H(2)$-unknotting number two (Corollaries 2.4 and 2.5).

The second one is a criterion on knots with $H(2)$-Gordian distance two by using a special value of the Jones polynomial (Theorem 3.1), which extends some criteria given in $[6,8]$.

As an application, we give tables of $H(2)$-Gordian distances between knots with up to 7 crossings (Tables 6 and 7). They improve the tables compiled in [6], where there remain 60 pairs of knots whose $H(2)$-Gordian distances are unsettled. Among these pairs we decide the $H(2)$-Gordian distances for 20 pairs of knots using the criteria above together with those given in [8]. Further, we can decide for 8 pairs of knots by virtue of the paper of Zeković [14]. She has given a new method for searching pairs of knots related by either a crossing change or an $H(2)$-move. Then she gave tables of pairs of knots with Gordian distance one and those with $H(2)$-Gordian distance one with at most 9 crossings.

This paper is organized as follows: In Sec. 2 we give a condition on the determinant of a knot or link which is obtained from an unknotting number one knot by a band surgery, which is deduced from Nakanishi's criterion. Then we give a condition for an unknotting number one knot to have $H(2)$-unknotting number two in terms of the determinant of a knot. In Sec. 3 we give a criterion of a pair of knots with $H(2)$-Gordian distance two using the special value of the Jones polynomial. In Sec. 4 we give the tables of the $H(2)$-Gordian distances between knots with up to seven crossings, which improves those in [6].

Notation. For knots and links we use Rolfsen notations [12, Appendix C]. For a knot or link $L$, we denote by $L$ ! its mirror image.

\section{Determinant of A Link OBTAINEd FROM AN UNKNOTTING NUMBER ONE KNOT BY A BAND SURGERY}

The following criterion is due to Nakanishi, which is implied from Proposition 13 in [11] and has been essentially given in Theorem 3 in [10]. 
Proposition 2.1. Let $K$ be an unknotting number one knot. If a knot $J$ is obtained from $K$ by a crossing change, then there exists an integer $s$ such that:

$$
\operatorname{det} J \equiv \pm s^{2} \quad(\bmod \operatorname{det} K)
$$

Using this proposition, we may deduce the following.

Theorem 2.2. Suppose that a knot or link $L$ is obtained from an unknotting number one knot $K$ by a coherent or incoherent band surgery. Then there exists an integer s such that:

$$
2 \operatorname{det} L \equiv \pm s^{2} \quad(\bmod \operatorname{det} K)
$$

The proof is similar to that of Theorem 4.2 in [8]. In order to prove this, we use the Jones polynomial [5]. We define the Jones polynomial $V(L ; t) \in \boldsymbol{Z}\left[t^{ \pm 1 / 2}\right]$ of an oriented link $L$ by the following formulas:

$$
\begin{gathered}
V(U ; t)=1 \\
t^{-1} V\left(L_{+} ; t\right)-t V\left(L_{-} ; t\right)=\left(t^{1 / 2}-t^{-1 / 2}\right) V\left(L_{0} ; t\right)
\end{gathered}
$$

where $U$ is the unknot and $L_{+}, L_{-}, L_{0}$ are three oriented links that are identical except near one point where they are as in Fig. 2 ; we call an ordered set $\left(L_{+}, L_{-}, L_{0}\right)$ a skein triple.

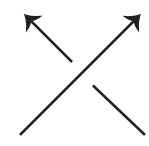

$L_{+}$

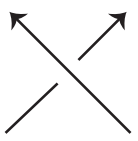

$L_{-}$

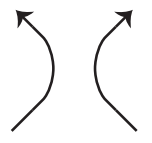

$L_{0}$

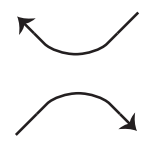

$L_{\infty}$

Figure 2. A skein triple $\left(L_{+}, L_{-}, L_{0}\right)$, and a knot $L_{\infty}$.

If $L_{+}$and $L_{-}$are knots, then $L_{0}$ is a 2-component link and we may consider another knot $L_{\infty}$ which is of the diagram of Fig. 2 . Then $L_{+} / L_{-}$and $L_{\infty}$ are related by an incoherent band surgery, and we have the following relation [3, Theorem 2]:

$$
V\left(L_{+} ; t\right)-t V\left(L_{-} ; t\right)+t^{3 \lambda}(t-1) V\left(L_{\infty} ; t\right)=0,
$$

where $\lambda$ is the linking number of $L_{0}$.

For a $c$-component link $L, i^{c-1} V(L ;-1)$ is an integer and the determinant $\operatorname{det} L$ is given by $\operatorname{det} L=|V(L ;-1)|$. Putting $t=-1$ in Eqs. (4) and (5), we obtain

$$
\begin{gathered}
-V\left(L_{+} ;-1\right)+V\left(L_{-} ;-1\right)=2 i V\left(L_{0} ;-1\right) ; \\
V\left(L_{+} ;-1\right)+V\left(L_{-} ;-1\right)=2(-1)^{\lambda} V\left(L_{\infty} ;-1\right) .
\end{gathered}
$$

Proof of Theorem 2.2. Suppose that a 2-component link $L$ is obtained from an unknotting number one knot $J$ by a coherent band surgery. Then there exists a knot $K$ such that $(J, K, L)$ is a skein triple; cf. Lemma 2.1(i) in [8]. Then by Proposition 2.1 there exists an integer $s$ with Eq. (1). From Eq. (6) we have $-V(J ;-1)+V(K ;-1)=2 i V(L ;-1)$, and so 
since $\operatorname{det} J=|V(J ;-1)|$ and $\operatorname{det} K=|V(K ;-1)|$, we obtain Eq. (2). For the case where $L$ and $J$ are related by an incoherent band surgery we use Eq. (7).

For an unknotting number one knot $K$ with $\operatorname{det} K \leq 115$ we list the impossible values of $\operatorname{det} L$, where $L$ is a knot or link with $\mathrm{d}_{2}(K, L)=1$ or $\mathrm{d}_{\mathrm{cb}}(K, L)=1$ in Tables 1 and 2. They are obtained by using the Mathematica program. Notice that if $K$ is a prime knot with up to 10 crossings, then $\operatorname{det} K \leq 111$. Therefore, Tables 1 and 2 yield Tables 3 and 4 . In Table 3 the symbol $\times$ means that the unknotting number one knots $J$ in the row and the knots $K$ in the column are not related by an incoherent band surgery, which also implies that the pairs $(J !, K !),(J, K !),(J !, K)$ are not related by an incoherent band surgery. For example, the pairs of knots $\left(6_{1}, 7_{4}\right),\left(6_{1} !, 7_{4} !\right),\left(6_{1}, 7_{4} !\right),\left(6_{1} !, 7_{4}\right)$ are not related by an incoherent band surgery. In Table 4 the symbol $\times$ means that the unknotting number one knots $J$ in the row and the oriented links $L$ in the column with any orientation are not related by a coherent band surgery, which implies that the pairs of oriented knots and links $(J, L),(J !, L !),(J, L !),(J !, L)$ with any orientation are not related by a coherent band surgery.

Example 2.3. $\mathrm{d}_{2}\left(5_{2}, 8_{17}\right)=\mathrm{d}_{2}\left(7_{1}, 8_{17}\right)=2$. Note that $8_{17}$ is negative-amphicheiral. First, by Table 3 we see $\mathrm{d}_{2}\left(5_{2}, 8_{17}\right)>1$ and $\mathrm{d}_{2}\left(7_{1}, 8_{17}\right)>1$. Notice that previously known methods in [6] (Theorems 4.1, 5.5, 8.1) and [8] (Theorems 4.2, 5.2(iii), 7.2) do not prove this; see Table 5. Conversely, we have

$$
\begin{aligned}
& \mathrm{d}_{2}\left(5_{2}, 8_{17}\right) \leq \mathrm{d}_{2}\left(5_{2}, 6_{3}\right)+\mathrm{d}_{2}\left(6_{3}, 8_{17}\right)=2 ; \\
& \mathrm{d}_{2}\left(7_{1}, 8_{17}\right) \leq \mathrm{d}_{2}\left(7_{1}, 4_{1}\right)+\mathrm{d}_{2}\left(4_{1}, 8_{17}\right)=2 .
\end{aligned}
$$

In fact, the knot $8_{17}$ is transformed into $4_{1}$ and $6_{3}$ by the $H(2)$-moves using the band and the crossing as shown in Fig. 3, respectively, which imply $\mathrm{d}_{2}\left(8_{17}, 4_{1}\right)=1$ and $\mathrm{d}_{2}\left(8_{17}, 6_{3}\right)=1$.

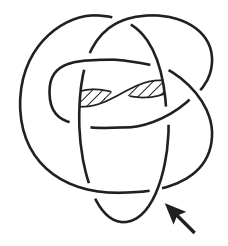

Figure 3 . The knot $8_{17}$ is transformed into $4_{1}$ and $6_{3}$ by $H(2)$-moves.

Since the $H(2)$-unknotting number of an unknotting number one knot is at most two by Theorem 3.1 in [7], Theorem 2.2 implies:

Corollary 2.4. Let $K$ be an unknotting number one knot and let $d=\operatorname{det} K$. Suppose that for any integer $x$

$$
x^{2} \not \equiv \pm 2 \quad(\bmod d),
$$

i.e., both 2 and -2 are quadratic non-residues modulo d. Then $\mathrm{u}_{2}(K)=2$. 
TABLE 1. Values for which $\operatorname{det} L$ does not take with $\mathrm{d}_{2}(K, L)=1$ or $\mathrm{d}_{\mathrm{cb}}(K, L)=1, K$ being an unknotting number one knot $(\mathrm{I})$.

\begin{tabular}{|c|c|}
\hline $\operatorname{det} K$ & $\operatorname{det} L \not \equiv$ \\
\hline $5,15,35,55,95,115$ & $1,4(\bmod 5)$ \\
\hline 9,27 & $3,6(\bmod 9)$ \\
\hline $13,39,91$ & $1,3,4,9,10,12(\bmod 13)$ \\
\hline 17,51 & $3,5,6,7,10,11,12,14(\bmod 17)$ \\
\hline 21 & $1,4,5,16,17,20(\bmod 21)$ \\
\hline 25,75 & $1,4,5,6,9,10,11,14,15,16,19,20,21,24(\bmod 25)$ \\
\hline 29,87 & $1,4,5,6,7,9,13,16,20,22,23,24,25,28(\bmod 29)$ \\
\hline 33 & $5,7,10,13,14,19,20,23,26,28(\bmod 33)$ \\
\hline 37,111 & $\begin{array}{l}1,3,4,7,9,10,11,12,16,21,25,26,27,28,30,33,34, \\
36(\bmod 37)\end{array}$ \\
\hline 41 & $\begin{array}{l}3,6,7,11,12,13,14,15,17,19,22,24,26,27,28,29,30, \\
34,35,38(\bmod 41)\end{array}$ \\
\hline 45 & $\begin{array}{l}1,3,4,6,9,11,12,14,15,16,19,21,24,26,29,30,31 \\
33,34,36,39,41,42,44(\bmod 45)\end{array}$ \\
\hline 49 & $7,14,21,28,35,42(\bmod 49)$ \\
\hline 53 & $\begin{array}{l}1,4,6,7,9,10,11,13,15,16,17,24,25,28,29,36,37 \\
38,40,42,43,44,46,47,49,52(\bmod 53)\end{array}$ \\
\hline 57 & $\begin{array}{l}5,10,11,13,17,20,22,23,26,31,34,35,37,40,44,46, \\
47,52(\bmod 57)\end{array}$ \\
\hline 61 & $\begin{array}{l}1,3,4,5,9,12,13,14,15,16,19,20,22,25,27,34,36 \\
39,41,42,45,46,47,48,49,52,56,57,58,60(\bmod 61)\end{array}$ \\
\hline 63 & $\begin{array}{l}1,3,4,5,6,12,15,16,17,20,21,22,24,25,26,30,33,37 \\
38,39,41,42,43,46,47,48,51,57,58,59,60,62(\bmod 63)\end{array}$ \\
\hline 65 & $\begin{array}{l}1,3,4,6,9,10,11,12,14,16,17,19,21,22,23,24,25 \\
26,27,29,30,31,34,35,36,38,39,40,41,42,43,44 \\
46,48,49,51,53,54,55,56,59,61,62,64(\bmod 65)\end{array}$ \\
\hline 69 & $\begin{array}{l}2,7,8,10,19,22,26,28,29,32,34,35,37,40,41,43,47 \\
50,59,61,62,67(\bmod 69)\end{array}$ \\
\hline
\end{tabular}

Let $d(0<d \leq 115)$ be an integer such that both 2 and -2 are quadratic non-residues modulo $d$. Then from Tables 1 and 2 we have:

$$
\begin{array}{r}
d=5,13,15,21,25,29,35,37,39,45,53,55,61,63,65, \\
\quad 69,75,77,85,87,91,93,95,101,105,109,111,115 .
\end{array}
$$

Consequently, the following unknotting number one knots have $H(2)$-unknotting number two; see $[1,2,7]$ for the table of $H(2)$-unknotting numbers of knots with up to nine crossings.

$$
\begin{aligned}
& 4_{1}, 6_{3}, 7_{7} ; 8_{l}, l=1,9,13,17,21 ; 9_{m}, m=2,12,14,24,30,33,39 ; \\
& 10_{n} ; n=9,10,18,26,32,33,59,60,71,82,84,88,95,104,107, \\
& 113,114,119,129,132,136,137,141,156,159,164 .
\end{aligned}
$$


TABLE 2. Values for which $\operatorname{det} L$ does not take with $\mathrm{d}_{2}(K, L)=1$ or $\mathrm{d}_{\mathrm{cb}}(K, L)=1, K$ being an unknotting number one knot (II).

\begin{tabular}{|c|c|}
\hline $\operatorname{det} K$ & $\operatorname{det} L \not \equiv$ \\
\hline 73 & $\begin{array}{l}5,7,10,11,13,14,15,17,20,21,22,26,28,29,30,31,33,34,39,40, \\
42,43,44,45,47,51,52,53,56,58,59,60,62,63,66,68(\bmod 73)\end{array}$ \\
\hline 77 & $\begin{array}{l}1,4,6,9,10,13,15,16,17,19,23,24,25,36,37,40,41,52,53,54, \\
58,60,61,62,64,67,68,71,73,76(\bmod 77)\end{array}$ \\
\hline 81 & $\begin{array}{l}3,6,12,15,21,24,27,30,33,39,42,48,51,54,57,60,66,69,75, \\
78(\bmod 81)\end{array}$ \\
\hline 85 & $\begin{array}{l}1,3,4,5,6,7,9,10,11,12,14,16,19,20,21,22,23,24,26,27,28,29, \\
31,34,36,37,39,40,41,44,45,46,48,49,51,54,56,57,58,59,61,62, \\
63,64,65,66,69,71,73,74,75,76,78,79,80,81,82,84(\bmod 85)\end{array}$ \\
\hline 89 & $\begin{array}{l}3,6,7,12,13,14,15,19,23,24,26,27,28,29,30,31,33,35,37,38,41,43, \\
46,48,51,52,54,56,58,59,60,61,62,63,65,66,70,74,75,76,77,82,83, \\
86(\bmod 89)\end{array}$ \\
\hline 93 & $\begin{array}{l}1,4,7,10,11,16,17,19,23,25,26,28,29,40,44,49,53,64,65,67,68 \\
70,74,76,77,82,83,86,89,92(\bmod 93)\end{array}$ \\
\hline 97 & $\begin{array}{l}5,7,10,13,14,15,17,19,20,21,23,26,28,29,30,34,37,38,39,40,41,42, \\
45,46,51,52,55,56,57,58,59,60,63,67,68,69,71,74,76,77,78,80,82, \\
83,84,87,90,92(\bmod 97)\end{array}$ \\
\hline 99 & $\begin{array}{l}3,5,6,7,10,12,13,14,15,19,20,21,23,24,26,28,30,33,38,39,40 \\
42,43,46,47,48,51,52,53,56,57,59,60,61,66,69,71,73,75,76,78 \\
79,80,84,85,86,87,89,92,93,94,96(\bmod 99)\end{array}$ \\
\hline 101 & $\begin{array}{l}1,4,5,6,9,13,14,16,17,19,20,21,22,23,24,25,30,31,33,36,37 \\
43,45,47,49,52,54,56,58,64,65,68,70,71,76,77,78,79,80,81,82, \\
84,85,87,88,92,95,96,97,100(\bmod 101)\end{array}$ \\
\hline 105 & $\begin{array}{l}1,4,5,6,9,11,14,16,17,19,20,21,22,24,25,26,29,31,34,36,37,38 \\
39,41,43,44,46,47,49,51,54,56,58,59,61,62,64,66,67,68,69,71, \\
74,76,79,80,81,83,84,85,86,88,89,91,94,96,99,100,101,104 \quad(\bmod 105)\end{array}$ \\
\hline 109 & $\begin{array}{l}1,3,4,5,7,9,12,15,16,20,21,22,25,26,27,28,29,31,34,35,36,38 \\
43,45,46,48,49,60,61,63,64,66,71,73,74,75,78,80,81,82,83,84 \\
87,88,89,93,94,97,100,102,104,105,106,108(\bmod 109)\end{array}$ \\
\hline 113 & $\begin{array}{l}3,5,6,10,12,17,19,20,21,23,24,27,29,33,34,35,37,38,39,40,42,43,45, \\
46,47,48,54,55,58,59,65,66,67,68,70,71,73,74,75,76,78,79,80,84,86, \\
89,90,92,93,94,96,101,103,107,108,110(\bmod 113)\end{array}$ \\
\hline
\end{tabular}

The numbers in (11) have a divisor congruent to 5 modulo 8. Indeed, using Lemma 2.6 below, Corollary 2.4 is restated as folllows.

Corollary 2.5. Let $K$ be an unknotting number one knot. If the determinant of $K$ is a multiple of $8 k+5$ for some $k, k=0,1,2, \ldots$, then $\mathrm{u}_{2}(K)=2$.

Lemma 2.6. A positive odd integer $d$ is a multiple of $8 k+5$ for some $k, k=0,1,2, \ldots$ if and only if both 2 and -2 are quadratic non-residues modulo $d$. 
TABle 3 . Knots $J$ and $K$ with $\mathrm{d}_{2}(J, K)>1, J$ being of unknotting number one.

\begin{tabular}{|c|c|c|c|c|c|c|c|c|}
\hline \multirow[b]{2}{*}{$K$} & \multirow[b]{2}{*}{$\operatorname{det} K$} & \multicolumn{7}{|c|}{$J$} \\
\hline & & $\begin{array}{c}4_{1} \\
8_{21}\end{array}$ & $\begin{array}{c}6_{1} \\
8_{11} \\
8_{20}\end{array}$ & $\begin{array}{l}6_{3} \\
8_{1}\end{array}$ & $7_{7}$ & 89 & 813 & 817 \\
\hline$U$ & 1 & $\times$ & & $x$ & $\times$ & $x$ & $x$ & $x$ \\
\hline $3_{1}, 8_{19}$ & 3 & & $x$ & $\times$ & & & & $x$ \\
\hline $4_{1}, 5_{1}$ & 5 & & & & $\times$ & $x$ & $\times$ & \\
\hline $5_{2}, 7_{1}$ & 7 & & & & & & $\times$ & $\times$ \\
\hline $6_{1}, 3_{1} \# 3_{1}, 8_{20}$ & 9 & $x$ & & $x$ & & $\times$ & $\times$ & $\times$ \\
\hline $6_{2}, 7_{2}$ & 11 & $\times$ & & & & $\times$ & & $\times$ \\
\hline $6_{3}, 7_{3}, 8_{1}$ & 13 & & & & & & $x$ & \\
\hline $7_{4}, 3_{1} \# 4_{1}, 8_{21}, 3_{1} \# 5_{1}$ & 15 & & $x$ & & & $x$ & & \\
\hline $7_{5}, 8_{2}, 8_{3}$ & 17 & & & $x$ & $\times$ & & & \\
\hline $7_{6}, 8_{4}$ & 19 & $x$ & & & & $\times$ & & \\
\hline $7_{7}, 8_{5}, 3_{1} \# 5_{2}$ & 21 & $\times$ & $x$ & & & $\times$ & & $x$ \\
\hline $8_{6}, 8_{7}$ & 23 & & & $\times$ & & & $\times$ & \\
\hline $8_{8}, 8_{9}, 4_{1} \# 4_{1}$ & 25 & & & $x$ & $x$ & & $x$ & $x$ \\
\hline $8_{10}, 8_{11}$ & 27 & & & $\times$ & & & & $x$ \\
\hline $8_{12}, 8_{13}$ & 29 & $\times$ & & $x$ & & $x$ & & \\
\hline $8_{14}$ & 31 & $x$ & & & & $x$ & & \\
\hline $8_{15}$ & 33 & & $\times$ & & & & $\times$ & $\times$ \\
\hline $8_{16}$ & 35 & & & $x$ & & $\times$ & $\times$ & \\
\hline \multirow[t]{4}{*}{$8_{17}$} & 37 & & & & $\times$ & & & \\
\hline & 39 & $\times$ & $x$ & & & $\times$ & & \\
\hline & 41 & $\times$ & & & $\times$ & $\times$ & & $\times$ \\
\hline & 43 & & & $\times$ & $x$ & & & \\
\hline $8_{18}$ & 45 & & & & & $\times$ & $\times$ & \\
\hline
\end{tabular}

In order to prove Lemma 2.6 we use the Jacobi symbol; cf. [4]. For an integer $m$ and an odd prime number $p$ the Legendre symbol $(m / p)$ is defined by

$$
\left(\frac{m}{p}\right)= \begin{cases}1 & \text { if } m \text { is a quadratic residue modulo } p \text { and } m \neq \equiv 0 \quad(\bmod p) \\ -1 & \text { if } m \text { is a quadratic non-residue modulo } p\end{cases}
$$

For an odd positive integer $n$ with prime factorization $n=p_{1} p_{2} \cdots p_{r}$, where $p_{i}$ is a prime number, the Jacobi symbol $(m / n)$ is defined by

$$
\left(\frac{m}{n}\right)=\prod_{i=1}^{r}\left(\frac{m}{p_{i}}\right)
$$

where $\left(m / p_{i}\right)$ is the Legendre symbol.

If $p_{i}$ is a quadratic residue modulo $n$ for each $i$, then by the Chinese remainder theorem $m$ is a quadratic residue modulo $n$. If $m$ is a quadratic residue modulo $n$, then the Jacobi 
TABLE 4. 2-component links $L$ and unknotting number one knots $J$ with $\mathrm{d}_{\mathrm{cb}}(J, L)>1$.

\begin{tabular}{|c|c|c|c|c|c|c|c|c|}
\hline \multirow[b]{2}{*}{$L$} & \multirow[b]{2}{*}{$\operatorname{det} L$} & \multicolumn{7}{|c|}{$J$} \\
\hline & & & $\begin{array}{c}6_{1} \\
8_{11} \\
8_{20}\end{array}$ & $\begin{array}{l}6_{3} \\
8_{1}\end{array}$ & $7_{7}$ & 89 & $8_{13}$ & 817 \\
\hline$\overline{U^{2}}$ & 0 & & & & & & & \\
\hline$H_{-}\left(=2_{1}^{2}\right)$ & 2 & & & & & & & \\
\hline$T_{4}=4_{1}^{2}, 7_{7}^{2}$ & 4 & $x$ & & $\times$ & $\times$ & $x$ & $\times$ & $x$ \\
\hline $3_{1} \# H_{-}, T_{6}\left(=6_{1}^{2}\right)$ & 6 & $x$ & $x$ & & & $\times$ & $x$ & \\
\hline $5_{1}^{2}, 7_{8}^{2}, T_{8}\left(=8_{1}^{2}\right), 8_{15}^{2}$ & 8 & & & & & & & \\
\hline $6_{2}^{2}, 4_{1} \# H_{-}, 5_{1} \# H_{-}$ & 10 & & & $\times$ & & $x$ & & $x$ \\
\hline $6_{3}^{2}, 3_{1} \# T_{4}, 8_{16}^{2}$ & 12 & & $\times$ & $\times$ & & & & $x$ \\
\hline $7_{1}^{2}, 5_{2} \# H_{-}$ & 14 & $x$ & & $\times$ & & $x$ & & \\
\hline $7_{3}^{2}, 7_{4}^{2}, 8_{2}^{2}, 8_{12}^{2}$ & 16 & $x$ & & $\times$ & $\times$ & $x$ & $\times$ & $\times$ \\
\hline $7_{2}^{2}$ & 18 & & & & & & & \\
\hline $7_{5}^{2}, 8_{6}^{2}$ & 20 & & & & $\times$ & $x$ & $\times$ & \\
\hline $8_{3}^{2}$ & 22 & & & $\times$ & $x$ & & $x$ & \\
\hline $7_{6}^{2}, 8_{4}^{2}$ & 24 & $x$ & $\times$ & & & $x$ & $x$ & \\
\hline $8_{5}^{2}$ & 26 & $x$ & & & $x$ & $x$ & & $x$ \\
\hline $8_{9}^{2}, 8_{11}^{2}$ & 28 & & & & & & $x$ & $x$ \\
\hline $8_{7}^{2}$ & 30 & & $\times$ & $\times$ & & $x$ & $\times$ & $\times$ \\
\hline $8_{10}^{2}, 8_{12}^{2}$ & 32 & & & & & & & \\
\hline $8_{8}^{2}$ & 34 & $x$ & & & & $x$ & $\times$ & $x$ \\
\hline \multirow[t]{2}{*}{$8_{14}^{2}$} & 36 & $x$ & & $\times$ & & $\times$ & $x$ & $x$ \\
\hline & 38 & & & $\times$ & $\times$ & & $\times$ & $\times$ \\
\hline $8_{13}^{2}$ & 40 & & & $\times$ & & $\times$ & & $x$ \\
\hline
\end{tabular}

TABLE 5. Invariants of the knots $5_{2}, 7_{1}$, and $8_{17}$.

\begin{tabular}{c|rrrr}
\hline$K$ & $\sigma(K)$ & $V(K ;-1)$ & $\operatorname{Arf}(K)$ & $\mathrm{u}_{2}(K)$ \\
\hline $5_{2}$ & 2 & -7 & 0 & 1 \\
$7_{1}$ & 6 & -7 & 0 & 1 \\
$8_{17}$ & 0 & 37 & 1 & 2 \\
\hline
\end{tabular}

symbol $(m / n)$ is 1 . We use the following formulas of the Jacobi symbol; cf. [13, p. 84].

$$
\begin{aligned}
\left(\frac{2}{n}\right) & =\left\{\begin{array}{lll}
1 & \text { if } n \equiv 1,7 & (\bmod 8) ; \\
-1 & \text { if } n \equiv 3,5 & (\bmod 8),
\end{array}\right. \\
\left(\frac{-2}{n}\right) & =\left\{\begin{array}{lll}
1 & \text { if } n \equiv 1,3 & (\bmod 8) \\
-1 & \text { if } n \equiv 5,7 & (\bmod 8)
\end{array}\right.
\end{aligned}
$$


Proof of Lemma 2.6. If $d$ is a multiple of $8 k+5$ for some $k, k=0,1,2, \ldots$, then both 2 and -2 are quadratic non-residues modulo $d$. In fact, the Jacobi symbols $( \pm 2 /(8 k+5))$ are -1 by Eqs. (15) and (16).

Suppose that any divisor of $d$ is not congruent to 5 modulo 8 . Let $d=p_{1} p_{2} \ldots p_{m}$ be a prime factorization; each $p_{i}$ is an odd prime number. Then we have two cases:

(i) $p_{i} \equiv 1,3(\bmod 8)$ for each $i$;

(ii) $p_{i} \equiv 1,7(\bmod 8)$ for each $i$.

In fact, if $p_{i} \equiv 3, p_{j} \equiv 7(\bmod 8)$, then $p_{i} p_{j} \equiv 5(\bmod 8)$. Then in Case (i) by Eq. $(16)$ -2 is a quadratic residue modulo $p_{i}$ for each $i$, and so -2 is a quadratic residue modulo $d$. Similarly, in Case (ii) 2 is a quadratic residue modulo $d$. This completes the proof.

\section{Criterion on knots with $H(2)$-Gordian distance two}

In [6] we have compiled a table of $H(2)$-Gordian distances between knots with up to seven crossings, where we use several criteria to give lower bounds. In [8] we gave further criteria for giving a lower bound of the $H(2)$-Gordian distance. In Theorems 7.1 and 7.3 in [6], the individual 6 pairs of knots are proved to have $H(2)$-Gordian distance two by using the special value of the Jones polynomial, which is generalized to Theorem 5.2(iii) in [8].

We give a further criterion of a pair of knots with $H(2)$-Gordian distance two:

Theorem 3.1. Let $K$ and $K^{\prime}$ be knots with $\mathrm{d}_{2}\left(K, K^{\prime}\right)=2$ and $V(K ; \omega)=V\left(K^{\prime} ; \omega\right)=$ $\pm(i \sqrt{3})^{\delta}$. If either

(i) $\sigma(K)-\sigma\left(K^{\prime}\right) \equiv 0(\bmod 8)$ and $\operatorname{Arf}(K) \neq \operatorname{Arf}\left(K^{\prime}\right)$, or

(ii) $\sigma(K)-\sigma\left(K^{\prime}\right) \equiv 4(\bmod 8)$ and $\operatorname{Arf}(K)=\operatorname{Arf}\left(K^{\prime}\right)$,

then

$$
V(K ;-1) \equiv V\left(K^{\prime} ;-1\right) \quad\left(\bmod 3^{\delta+1}\right) .
$$

Proof. Let $J$ be a knot which is obtained from both $K$ and $K^{\prime}$ by an $H(2)$-move; $\mathrm{d}_{2}(J, K)=$ $\mathrm{d}_{2}\left(J, K^{\prime}\right)=1$. First, we show $\sigma(J) \equiv \sigma(K) \pm 2(\bmod 8)$. Indeed, if we assume $\sigma(J) \equiv \sigma(K)$ $(\bmod 4)$, then by Lemma 6.1 in $[6]$ we have:

- If $\sigma(K)-\sigma\left(K^{\prime}\right) \equiv 0(\bmod 8)$, then $\operatorname{Arf}(K)=\operatorname{Arf}\left(K^{\prime}\right)$.

- If $\sigma(K)-\sigma\left(K^{\prime}\right) \equiv 4(\bmod 8)$, then $\operatorname{Arf}(K) \neq \operatorname{Arf}\left(K^{\prime}\right)$.

This contradicts our assumption.

Next, we show $V(J ; \omega)= \pm v_{0}$, where $v_{0}=V(K ; \omega)=V\left(K^{\prime} ; \omega\right)$. By [6, Theorem 5.3] we have $V(J ; \omega) \in\left\{ \pm v_{0}, \pm i \sqrt{3}^{ \pm 1} v_{0}\right\}$. Assume $V(J ; \omega) / v_{0}=\epsilon i \sqrt{3}, \epsilon= \pm 1$. Then by Theorem 5.5 in $[6]$ we obtain:

(a) If $\sigma(J)-\sigma(K) \equiv 2 \epsilon(\bmod 8)$, then $\operatorname{Arf}(J)=\operatorname{Arf}(K)$.

(b) If $\sigma(J)-\sigma(K) \equiv-2 \epsilon(\bmod 8)$, then $\operatorname{Arf}(J) \neq \operatorname{Arf}(K)$.

(c) If $\sigma(J)-\sigma\left(K^{\prime}\right) \equiv 2 \epsilon(\bmod 8)$, then $\operatorname{Arf}(J)=\operatorname{Arf}\left(K^{\prime}\right)$.

(d) If $\sigma(J)-\sigma\left(K^{\prime}\right) \equiv-2 \epsilon(\bmod 8)$, then $\operatorname{Arf}(J) \neq \operatorname{Arf}\left(K^{\prime}\right)$.

Therefore, $K$ and $K^{\prime}$ do not satisfy the conditions (i) nor (ii). Similarly, we may prove $V(J ; \omega) \neq \pm i \sqrt{3}^{-1} v_{0}$. 
Thus we have $V(J ; \omega)=\eta v_{0}, \eta= \pm 1$. Then by Theorem 5.2(iii) in [8] we obtain $\eta V(J ;-1) \equiv V(K ;-1) \equiv V\left(K^{\prime} ;-1\right)\left(\bmod 3^{\delta+1}\right)$, completing the proof.

Example 3.2. Let $K=66_{1}$ ! and $K^{\prime}=7_{7}$. Then $V(K ; \omega)=V\left(K^{\prime} ; \omega\right)=-i \sqrt{3}, \sigma(K)=$ $\sigma\left(K^{\prime}\right)=0, \operatorname{Arf}(K)=0, \operatorname{Arf}\left(K^{\prime}\right)=1$, and $9=V(K ;-1) \not \equiv V\left(K^{\prime} ;-1\right)=21(\bmod 9)$. Thus by Theorem 3.1 we obtain $\mathrm{d}_{2}\left(K, K^{\prime}\right) \neq 2$, which implies $\mathrm{d}_{2}\left(K, K^{\prime}\right)=3$ by [6, Table 3$]$.

\section{H(2)-Gordian distances of KNOts With up to SEven CROSSINGS}

In Tables 6 and 7 we list the $H(2)$-Gordian distances between knots with up to seven crossings, which improves those in [6], where the meanings of the marks are as follows:

- The marks $1-2,2-3,1-3$ mean 1 or 2,2 or 3,1 or 2 or 3 , respectively.

- The mark $1^{\mathrm{z})}$ means that the distance is confirmed to be 1 by Fig. 20 in [14], and $2^{\mathrm{z})}$ means that the distance is decided to be 2 from the inequality $\mathrm{d}_{2}\left(7_{1}, 7_{7}\right) \leq$ $\mathrm{d}_{2}\left(7_{1}, 6_{3}\right)+\mathrm{d}_{2}\left(6_{3}, 7_{7}\right)=2$.

- The mark $2^{\mathrm{m})}$ means that the distance is decided to be 2 by using Theorem 4.2 (Example 4.5) in [8].

- The mark $2^{\mathrm{mn})}$ means that the distance is decided to be 2 by using either by Theorem 4.2 (Example 4.5) in [8] or Theorem 2.2 (Table 3).

- The mark $2^{\mathrm{v})}$ means that the distance is decided to be 2 by using Theorem 5.8 (Example 5.10) in [8].

- The mark $2^{\text {mnv }}$ means that the distance is decided to be 2 by using by either Theorem 4.2 (Example 4.5) in [8], Theorem 2.2 (Table 3), or Theorem 5.8 (Example 5.10) in $[8]$.

- The mark $3^{\mathrm{v})}$ means that the distance is decided to be 3 by using Theorem 3.1 (Example 3.2).

\section{ACKnowledgements}

The author was partially supported by KAKENHI, Grant-in-Aid for Scientific Research (C) (No. 21540092), Japan Society for the Promotion of Science. He also would like to thank Professor Masaaki Furusawa for his suggestion for the proof of Lemma 2.6.

\section{REFERENCES}

1. Tetsuya Abe and Taizo Kanenobu, Unoriented band surgery on knots and links, Kobe J. Math. 31 (2014), no. 1-2, 21-44. MR 3307286

2. Yuanyuan Bao, H(2)-unknotting operation related to 2-bridge links, Topology Appl. 159 (2012), no. 8, 2158-2167. MR 2902750

3. Joan S. Birman and Taizo Kanenobu, Jones' braid-plat formula and a new surgery triple, Proc. Amer. Math. Soc. 102 (1988), no. 3, 687-695. MR 929004 (89c:57003)

4. Kenneth Ireland and Michael Rosen, A classical introduction to modern number theory, second ed., Graduate Texts in Mathematics, vol. 84, Springer-Verlag, New York, 1990. MR 1070716 (92e:11001)

5. V. F. R. Jones, Hecke algebra representations of braid groups and link polynomials, Ann. of Math. (2) 126 (1987), no. 2, 335-388. MR 908150 (89c:46092)

6. Taizo Kanenobu, H(2)-Gordian distance of knots, J. Knot Theory Ramifications 20 (2011), no. 6, 813-835. MR 2812265 (2012e:57011) 
TABLE 6. $H(2)$-Gordian distances of knots with up to 6 crossings.

\begin{tabular}{l|ccccccccc} 
& $3_{1}$ & 4 & $5_{1}$ & $5_{2}$ & $6_{1}$ & $6_{2}$ & $6_{3}$ & $3_{1} \# 3_{1}$ & $3_{1} ! \# 3_{1}$ \\
\hline$U$ & 1 & 2 & 1 & 1 & 1 & 1 & 2 & 2 & 2 \\
$3_{1}$ & 0 & 1 & 2 & 2 & 2 & 1 & 2 & 1 & 1 \\
$3_{1} !$ & 2 & 1 & 2 & 1 & 2 & 2 & 2 & 1 & 1 \\
$4_{1}$ & & 0 & $2-3$ & 1 & 2 & 2 & 1 & 2 & 2 \\
$5_{1}$ & & & 0 & 2 & $1-2$ & 1 & 2 & 3 & 2 \\
$5_{1} !$ & & & $1^{\text {z) }}$ & 2 & 1 & $1-2$ & 2 & 3 & 2 \\
$5_{2}$ & & & & 0 & 1 & 2 & 1 & 2 & 2 \\
$5_{2} !$ & & & & 2 & 2 & 1 & 1 & 2 & 2 \\
$6_{1}$ & & & & & 0 & $1-2$ & 2 & $2-3$ & 1 \\
$6_{1} !$ & & & & & $1-2$ & 2 & 2 & $2-3$ & 1 \\
$6_{2}$ & & & & & & 0 & 1 & 2 & 2 \\
$6_{2} !$ & & & & & & 2 & 1 & 2 & 2 \\
$6_{3}$ & & & & & & & 0 & 2 & 3 \\
$3_{1} \# 3_{1}$ & & & & & & & 0 & 2 \\
$3_{1} ! \# 3_{1} !$ & & & & & & & $1-2$ & 2 \\
$3_{1} ! \# 3_{1}$ & & & & & & & & 2 & 0
\end{tabular}

7. Taizo Kanenobu and Yasuyuki Miyazawa, H(2)-unknotting number of a knot, Commun. Math. Res. 25 (2009), no. 5, 433-460. MR 2573402 (2010i:57019)

8. Taizo Kanenobu and Hiromasa Moriuchi, Links which are related by a band surgery or crossing change, Bol. Soc. Mat. Mex. (3) 20 (2014), no. 2, 467-483. MR 3264627

9. Hitoshi Murakami, Some metrics on classical knots, Math. Ann. 270 (1985), no. 1, 35-45.

10. Yasutaka Nakanishi, A note on unknotting number. II, J. Knot Theory Ramifications 14 (2005), no. 1, 3-8. MR 2124552 (2006b:57006)

11. Yasutaka Nakanishi and Yuki Okada, Differences of Alexander polynomials for knots caused by a single crossing change, Topology Appl. 159 (2012), no. 4, 1016-1025. MR 2876708 (2012m:57014)

12. Dale Rolfsen, Knots and links, Mathematics Lecture Series, vol. 7, Publish or Perish, Inc., Houston, TX, 1990, Corrected reprint of the 1976 original. MR 1277811 (95c:57018)

13. Teiji Takagi, Shotou Seisûron Kougi [Lectures on elementary number theory], second ed., Kyoritsu Shuppan, Tokyo, 1971, Japanese.

14. Ana Zeković, Computation of Gordian distances and $\mathrm{H}_{2}$-Gordian distances of knots, Yugosl. J. Oper. Res. 25 (2015), no. 1, 133-152. MR 3331990

Department of Mathematics, Osaka City University, Sugimoto, Sumiyoshi-ku, Osaka 5588585, JAPAN

E-mail address: kanenobu@sci.osaka-cu.ac.jp 
TABLE 7. $H(2)$-Gordian distances of knots with up to 7 crossings.

\begin{tabular}{|c|c|c|c|c|c|c|c|c|}
\hline & $7_{1}$ & $7_{2}$ & $7_{3}$ & $7_{4}$ & $7_{5}$ & $7_{6}$ & $7_{7}$ & $3_{1} \# 4_{1}$ \\
\hline$U$ & 1 & 1 & 1 & 1 & 2 & 1 & 2 & 1 \\
\hline $3_{1}$ & 1 & 1 & 1 & 2 & 1 & 1 & 2 & 2 \\
\hline $3_{1} !$ & 2 & 2 & 1 & 2 & 1 & 2 & 1 & 2 \\
\hline $4_{1}$ & 1 & 2 & 2 & 1 & 1 & 2 & 2 & 1 \\
\hline $5_{1}$ & 2 & $1^{\mathrm{z})}$ & 2 & 2 & 2 & 1 & 2 & 2 \\
\hline $5_{1} !$ & 2 & 1 & 2 & 2 & 2 & $1-2$ & 2 & 2 \\
\hline $5_{2}$ & 2 & 2 & $2^{\mathrm{m})}$ & 2 & 1 & 2 & 1 & 2 \\
\hline $5_{2} !$ & $1^{\mathrm{z})}$ & $1-2$ & $2^{\mathrm{m})}$ & 2 & 1 & 1 & 2 & 2 \\
\hline $6_{1}$ & 2 & 1 & 1 & $2^{\mathrm{mnv})}$ & 2 & 2 & 2 & 2 \\
\hline $6{ }_{1} !$ & $1-2$ & 2 & 1 & $2^{\mathrm{mnv})}$ & 2 & 1 & $3^{\mathrm{v})}$ & 2 \\
\hline $6_{2}$ & $1-2$ & $1^{\mathrm{z})}$ & $2^{\mathrm{m})}$ & 1 & 1 & 1 & 1 & 2 \\
\hline $62 !$ & 2 & 2 & $2^{\mathrm{m})}$ & 2 & 1 & 2 & 2 & $1-2$ \\
\hline $6_{3}$ & $1^{z)}$ & $1-2$ & $2-3$ & 2 & $2^{\mathrm{mn})}$ & 1 & 1 & 2 \\
\hline $3_{1} \# 3_{1}$ & 2 & 2 & 2 & 1 & 2 & 2 & 1 & 1 \\
\hline $3_{1}$ !\#3 $3_{1}$ ! & 2 & 2 & 2 & $1-3$ & 2 & 2 & $1-2$ & $1-3$ \\
\hline $3_{1} ! \# 3_{1}$ & 2 & 2 & 2 & $1-2$ & 2 & 2 & 2 & 1 \\
\hline $7_{1}$ & 0 & $1-2$ & $2^{\mathrm{m})}$ & 2 & $1-2$ & $1-2$ & $2^{\mathrm{z})}$ & 2 \\
\hline $7_{1} !$ & 2 & 2 & $2^{\mathrm{m})}$ & 2 & $1^{\mathrm{z})}$ & 2 & $1^{\mathrm{z})}$ & 2 \\
\hline $7_{2}$ & & 0 & $2^{\mathrm{m})}$ & $1-2$ & $1-2$ & $1-2$ & $1-2$ & 2 \\
\hline $7_{2} !$ & & 2 & $2^{\mathrm{m})}$ & 2 & $1-2$ & 2 & 2 & 1 \\
\hline $7_{3}$ & & & 0 & 2 & 2 & $2^{\mathrm{m})}$ & 2 & 2 \\
\hline $7_{3} !$ & & & $1-2$ & 2 & 2 & $2^{\mathrm{m})}$ & 2 & 2 \\
\hline $7_{4}$ & & & & 0 & 2 & $1-2$ & 1 & 2 \\
\hline $7_{4} !$ & & & & 2 & 2 & 2 & $2^{\mathrm{v})}$ & $1-2$ \\
\hline $7_{5}$ & & & & & 0 & $2^{\mathrm{m})}$ & $2^{\mathrm{mn})}$ & 2 \\
\hline $7_{5} !$ & & & & & $1-2$ & $2^{\mathrm{m})}$ & $2^{\mathrm{mn})}$ & 2 \\
\hline $7_{6}$ & & & & & & 0 & 2 & 2 \\
\hline $7_{6} !$ & & & & & & 2 & 1 & 1 \\
\hline $7_{7}$ & & & & & & & 0 & $2^{\mathrm{v})}$ \\
\hline $7_{7} !$ & & & & & & & 2 & $1-2$ \\
\hline $3_{1} ! \# 4_{1}$ & & & & & & & & 2 \\
\hline
\end{tabular}

